\title{
Association of loss of heterozygosity with cytogenetic abnormalities in acute myeloid leukemia and myelodysplastic syndrome
}

\author{
R.F. Pinheiro' ${ }^{1}$, F.M. Serioํ, M.R.R. Silva ${ }^{1}$, M.R.S. Briones ${ }^{3}$ and M.L.L.F. Chauffaille ${ }^{1}$ \\ ${ }^{1}$ Disciplina de Hematologia e Hemoterapia, ${ }^{2}$ Disciplina de Anatomia Patológica, ${ }^{3}$ Departamento de \\ Microbiologia, Imunologia e Parasitologia, Escola Paulista de Medicina, Universidade Federal de São \\ Paulo, São Paulo, SP, Brasil
}

Correspondence to: M.L.L.F. Chauffaille, Hematologia, EPM, UNIFESP, Rua Botucatu, $740,3^{\circ}$ andar, 04023-900 São Paulo, SP, Brasil

Fax: +55-11-5571-8806. E-mail: chauffaill@hemato.epm.br

\begin{abstract}
Deletions on chromosomes 5 and 7 are frequently seen in myelodysplastic syndrome (MDS) and acute myeloid leukemia (AML). It is assumed that these deletions indicate loss of tumor suppressor genes on these chromosomes and until these tumor suppressor genes are identified, the functional consequences of these deletions and the molecular basis of these myeloid disorders cannot be completely understood. We evaluated loss of heterozygosity (LOH) in 44 patients (18 MDS and 26 AML, diagnosed according to WHO classification criteria) at diagnosis, using a four-microsatellite marker panel: an intragenic marker on the 7th intron of gene IRF-1 of the $5 \mathrm{q} 31.1$ region and three markers located inside the $7 \mathrm{q} 31.1$ region and correlated the LOH with karyotype abnormalities. The microsatellites chosen corresponded to chromosome regions frequently deleted in MDS/ AML. The samples with $Q$ (peak area) less than or equal to 0.50 were indicative of $L O H$. The percent of informative samples (i.e., heterozygous) for the intragenic microsatellite in gene IRF-1 and in loci D7S486, D7S515 and D7S522 were 66.6, 73.7, 75.5, and $48.8 \%$, respectively. Cytogenetic abnormalities by G-banding were found in 36\% (16/44) of the patients (2 of 18 MDS and 14 of 26 AML patients). We found a significantly positive association of the occurrence of LOH with abnormal karyotype $(P<0.05$; chisquare test) and there were cases with LOH but the karyotype was normal (by G-banding). These data indicate that LOH in different microsatellite markers is possibly an event previous to chromosomal abnormalities in these myeloid neoplasias.
\end{abstract}

Key words: Myelodysplastic syndrome; Acute myeloid leukemia; Cytogenetic abnormalities; Loss of heterozygosity

Publication supported by FAPESP.

Received January 31, 2008. Accepted July 2, 2008

Loss of heterozygosity ( $\mathrm{LOH}$ ) describes the homozygous state of a distinct chromosomal region and points to the presence of closely located inactivated tumor suppressor gene (TSG) that may be involved in the malignant transformation $(1,2)$. Loss of heterozygosity in tumor cells has been analyzed using microsatellite markers (2).

Cytogenetic abnormalities are important to identify malignant cells and to indicate prognosis in myeloid malignancies (3). Translocations, duplications, deletions, insertions, and inversions involve the repositioning, addition or omission of millions of nucleotides and are associated with genomic instability in cancer cells.

Myelodysplastic syndrome (MDS) and acute myeloid leukemia (AML) are myeloid malignant disorders of hematopoietic stem cells characterized by numerous cytogenetic abnormalities. Alterations on chromosome 5, which include interstitial deletions of the long arm or complete loss of the entire chromosome (monosomy 5), are frequently observed in MDS and AML patients (3). Deletion of chromosome 7q is also common in MDS and AML. Loss of whole chromosome 7 or deletion of the long arm are detected in up to $20 \%$ of patients with MDS or AML and the 
highest frequency is noted in MDS and AML arising after cytotoxic therapy (3). It is assumed that these deletions indicate loss of possible TSG in these chromosomes and until these TSG are identified, the functional consequences of these deletions and the molecular basis of these myeloid disorders cannot be completely understood.

Chromosome abnormalities are important markers of genetic instability but are considered to be late events during MDS transformation and AML evolution. The question is what are the initial steps in MDS and AML related to these abnormalities? Could $\mathrm{LOH}$ be a sign of future alterations in these cases?

The aim of this report is to evaluate $\mathrm{LOH}$ in MDS and AML patients using a four-microsatellite marker panel: an intragenic marker on the 7th intron of gene interferon regulatory factor 1 (IRF-1) of the $5 \mathrm{q} 31.1$ region and three markers located inside the 7q31.1 region and to correlate the LOH with karyotype abnormalities.

Karyotype analysis was performed according to standard techniques (4). When possible, 20 G-banded metaphases were analyzed and classified according to the International System for Human Cytogenetic Nomenclature (5). Favorable karyotype in MDS was considered to be $\operatorname{del}(20 q),-Y$, normal and $\operatorname{del}(5 q)$ isolated; unfavorable were complex karyotype (at least three abnormalities in metaphase) and abnormalities of chromosome 7 (6); intermediate were those not classified as favorable and unfavorable. Favorable karyotype in AML was: inv(16)/t(16;16), $\mathrm{t}(15 ; 17)$ and $\mathrm{t}(8 ; 21)$; intermediate was normal, +8, +6, -Y and del(12p), while unfavorable were alterations of chromosomes 3, 9, 11, 20, 21, del(5q), del(7q), -5, -7 and complex karyotype (7).

Paired samples of bone marrow and buccal smears were obtained, after informed consent, from 44 patients (18 MDS and $26 \mathrm{AML}$ ) at diagnosis. This study was approved by the Institutional Review Ethics Committee. The diagnoses of AML and MDS were made according to the World Health Organization (Table 1) (8).

Buccal smear collection was carried out with sterile cytology brushes and served as normal controls. Patients were instructed to brush the inside of both sides of the cheek for $30 \mathrm{~s}$, and the brush was kept in a sterile saline solution for extraction. Genomic DNA was extracted from buccal smears by the Puregene DNA isolation kit (Gentra Systems, USA) according to manufacturer recommendations.

Bone marrow samples were obtained for cytogenetic analysis and for DNA extraction by standard procedures (4). Bone marrow DNA was extracted by conventional cell lysis and proteinase $\mathrm{K}$ method. DNA was purified by phenol-chloroform extraction and precipitated with sodium acetate and ethanol (9). The concentration and quality of DNA were measured by the GeneQuant PRO RNA/DNA calculator (GE Healthcare, USA).

PCR was performed on $25 \mathrm{ng}$ DNA from bone marrow aspirates and buccal smears, in a $25 \mu \mathrm{L}$ containing $1 \mathrm{X}$ buffer (20 mM Tris- $\mathrm{HCl}, \mathrm{pH} 8.4,50 \mathrm{mM} \mathrm{KCl}$ ), $2 \mathrm{mM} \mathrm{MgCl}$, $200 \mu \mathrm{M}$ of each dNTP, $10 \mathrm{pmol}$ of each primer, and the forward primer was 5 ' end fluorescently labeled (FAM), $0.625 \mathrm{U}(0.12 \mu \mathrm{L})$ AmpliTaq Gold DNA polymerase (PE Applied Biosystems, USA) (9).

Amplification was performed in a Perkin Elmer 9700 Thermal Controller (PE Applied Biosystems) after an initial denaturation at $94^{\circ} \mathrm{C}$ for $12 \mathrm{~min}$. Twenty-five cycles were performed using the following temperature and time profile: denaturation at $94^{\circ} \mathrm{C}$ for $30 \mathrm{~s}$, annealing at $55^{\circ} \mathrm{C}$ for 30 $\mathrm{s}$, extension at $70^{\circ} \mathrm{C}$ for $30 \mathrm{~s}$, and a final extension of $72^{\circ} \mathrm{C}$ for $25 \mathrm{~min}$ to minimize stutter bands. The PCR products were analyzed on a $6 \%$ polyacrylamide gel in $1 \mathrm{X}$ Trisborate-EDTA buffer in the 377 automated DNA sequencer (PE Applied Biosystems). One microliter of each PCR product was combined with $4 \mu \mathrm{L}$ formamide, $0.5 \mu \mathrm{L}$ blue dextran, and $0.5 \mu \mathrm{L}$ of a fluorescent size marker (GS 500 ROX; PE Applied Biosystems). The mixture samples were heated to $90^{\circ} \mathrm{C}$ for $5 \mathrm{~min}$ and then cooled on ice until ready to load.

The electrophoresis conditions were $3000 \mathrm{~W}$ and $51^{\circ} \mathrm{C}$. PCR products were quantitatively detected by fragmentation analysis using $A B I$ GeneScan and $A B I$ Genotyper software (PE Applied Biosystems).

Cytogenetic abnormalities by G-banding were detected in $36 \%(16 / 44)$ of the patients, specifically 2 of $18 \mathrm{MDS}$ and 14 of 26 AML patients (Table 1).

The criteria selected for detecting allele loss were described by Cawkwell et al. (10). The samples with $Q$ (peak area) less than or equal to 0.50 indicated LOH. The percent of informative samples (i.e., heterozygous) for the intragenic microsatellite in gene IRF-1 and in loci D7S486, D7S515 and D7S522 were 66.6, 73.7, 75.5, and 48.8\%, respectively. The results of microsatellite analysis are summarized in Table 2.

Allelic loss occurred in 2 of $29(6 \%)$ informative cases for IRF-1. One patient (case 21) was an AML with prior MDS and the other (case 43) was an AML secondary to therapy, both patients had an unfavorable karyotype abnormality.

$\mathrm{LOH}$ was detected for one or more markers in 5 of 44 (11\%) patients. Cases 19 and 31 presented LOH for the 3 markers (D7S486, D7S515 and D7S522). Case 21 showed LOH for markers D7S486 and D7S515 and cases 5 and 23 presented LOH for marker D7S515. Among the 2 patients who presented deletion $5 q$ (cases 15 and 21 ), only case 21 
Table 1. Classification of patients in the present study according to WHO criteria (8) and results of karyotyping and microsatellite analyses.

\begin{tabular}{|c|c|c|c|}
\hline Patient No. & WHO classification & Cytogenetics & $\mathrm{LOH}$ \\
\hline 1 & Refractory cytopenia with multilineage dysplasia (RCMD) & Without metaphases & \\
\hline 2 & Refractory cytopenia with multilineage dysplasia (RCMD) & $46, X \times[20]$ & \\
\hline 3 & Refractory anemia with excess blasts- 1 (RAEB-1) & $46, X Y[20]$ & \\
\hline 4 & Refractory anemia with ringed sideroblasts (RARS) & $46, X Y[20]$ & \\
\hline 5 & Refractory cytopenia with multilineage dysplasia (RCMD) & $46, X X[20]$ & D7S515 \\
\hline 6 & Refractory anemia with excess blasts-2 (RAEB-2) & $46, X Y[20]$ & \\
\hline 7 & Refractory anemia with ringed sideroblasts (RARS) & $46, X X[20]$ & \\
\hline 8 & Refractory anemia (RA) & Without metaphases & \\
\hline 9 & Refractory anemia (RA) & $46, X Y[20]$ & \\
\hline 10 & Refractory anemia with ringed sideroblasts (RARS) & Without metaphases & \\
\hline 11 & Refractory anemia (RA) & $46, X Y[15]$ & \\
\hline 12 & Refractory anemia (RA) & Without metaphases & \\
\hline 13 & Refractory cytopenia with multilineage dysplasia (RCMD) & $46, X Y[20]$ & \\
\hline 14 & Refractory anemia with ringed sideroblasts (RARS) & Without metaphases & \\
\hline 15 & Refractory anemia with excess blasts-2 (RAEB-2) & $46, X X, \operatorname{del}(5)(q 31)[15]$ & \\
\hline 16 & Refractory anemia with excess blasts-2 (RAEB-2) & Condensed metaphases & \\
\hline 17 & Refractory cytopenia with multilineage dysplasia (RCMD) & $46, X \times[20]$ & \\
\hline 18 & Refractory anemia with excess blasts-1 (RAEB-1) & $47, X Y,+3[4] / 46, X Y[12]$ & \\
\hline 19 & AML with multilineage dysplasia with prior MDS & $45, X Y,-7[16] / 46, X Y[4]$ & D7S486, 515, 522 \\
\hline 20 & AML with multilineage dysplasia with prior MDS & $46, X Y[15]$ & \\
\hline 21 & AML with multilineage dysplasia with prior MDS & $\begin{array}{l}47, X X,+8[1] / 47, X X, \operatorname{del}(5)(q 13 q 33) \\
\operatorname{del}(7)(q 22),+8[2] / 49, X X, \operatorname{del} \\
(7)(q 22),+8,+11,+18[1]\end{array}$ & IRF-1, D7S486, 515 \\
\hline 22 & AML with multilineage dysplasia with prior MDS & $46, X \times[20]$ & \\
\hline 23 & AML with multilineage dysplasia without prior MDS & Without metaphases & D7S515 \\
\hline 24 & AML with multilineage dysplasia without prior MDS & $46, X X[20]$ & \\
\hline 25 & AML with multilineage dysplasia with prior MDS & $46, X X$, inc $[9]$ & \\
\hline 26 & AML with multilineage dysplasia with prior MDS & $46, X Y[20]$ & \\
\hline 27 & AML with multilineage dysplasia with prior MDS & $48, X X,+8,+9[10]$ & \\
\hline 28 & AML with multilineage dysplasia with prior MDS & $48, X Y,+14,+22[14] / 46, X Y[1]$ & \\
\hline 39 & AML not otherwise categorized/myelomonocytic & $46, X Y[8]$ & \\
\hline 30 & AML not otherwise categorized/monocytic & $46, X Y, t(1 ; 2)(p 31 ; q 34)[20]$ & \\
\hline 31 & AML not otherwise categorized/AML without maturation & $47, X Y,+8[16] / 46, X Y[4]$ & D7S486, 515, 522 \\
\hline 32 & AML not otherwise categorized/AML with maturation & $46, X Y[20]$ & \\
\hline 33 & $\begin{array}{l}\text { AML with recurrent cytogenetic translocations/AML with } \\
\mathrm{t}(8 ; 21)(\mathrm{q} 22 ; \mathrm{q} 22)\end{array}$ & $46, \mathrm{XY}, \mathrm{t}(8 ; 21)(\mathrm{q} 22 ; \mathrm{q} 22)[15]$ & \\
\hline 34 & $\begin{array}{l}\text { AML with recurrent cytogenetic translocations/AML with } \\
t(8 ; 21)(q 22 ; q 22)\end{array}$ & $46, \mathrm{XY}, \mathrm{t}(8 ; 21)(\mathrm{q} 22 ; \mathrm{q} 22)[20]$ & \\
\hline 35 & $\begin{array}{l}\text { AML with recurrent cytogenetic translocations/AML with } \\
t(15 ; 17)(q 22 ; q 12)\end{array}$ & $46, X X, t(15 ; 17)(q 22 ; q 11)[20]$ & \\
\hline 36 & AML not otherwise categorized/AML with maturation & $46, X X[20]$ & \\
\hline 37 & $\begin{array}{l}\text { AML with recurrent cytogenetic translocations/ AML with } \\
t(15 ; 17)(q 22 ; q 12)\end{array}$ & $46, X X, t(15 ; 17)(q 22 ; q 12)[20]$ & \\
\hline 38 & AML not otherwise categorized/AML without maturation & $47, X X, \operatorname{del}(7)(q 31),+8[3] / 46, X X[8]$ & \\
\hline 39 & AML not otherwise categorized/AML with maturation & $46, X Y[13]$ & \\
\hline 40 & AML not otherwise categorized/AML with maturation & Without metaphases & \\
\hline 41 & AML not otherwise categorized/megakaryocytic & $46, X X, \operatorname{add}(21)(q 22)[15]$ & \\
\hline 42 & AML not otherwise categorized/myelomonocytic & Without metaphases & \\
\hline 43 & AML therapy related & $\begin{array}{l}53-56, X X,+1,+8,+9,+10, i ?(11) \\
(q 13),+14,+15,+16,+17,+18,+19, \\
\sim 6 \mathrm{dmin}\end{array}$ & IRF-1 \\
\hline 44 & $\begin{array}{l}\text { AML with recurrent cytogenetic translocations/AML with } \\
t(15 ; 17)(q 22 ; q 12)\end{array}$ & $46, X Y, t(15 ; 17)(q 22 ; q 12)[20]$ & \\
\hline
\end{tabular}

$\mathrm{WHO}=$ World Health Organization; MDS = myelodysplastic syndrome; AML = acute myeloid leukemia; LOH = loss of heterozygosity. 
presented LOH for IRF-1. Among the 3 patients with deletion $7 q$ or monosomy 7 (cases 19, 21, and 38), two cases (19 and 21) presented $\mathrm{LOH}$ for at least one intragenic microsatellite of 7q31.1. Only 1 patient (case 5 ) with nor-

Table 2. Analysis of loss of heterozygosity (LOH) of the IRF-1 gene and the markers at $7 q$.

\begin{tabular}{|c|c|c|c|c|}
\hline \multirow[t]{2}{*}{ Patient No. } & \multirow[t]{2}{*}{$I R F-1$} & \multicolumn{3}{|c|}{ Markers at $7 q$} \\
\hline & & D7S486 & D7S515 & D7S522 \\
\hline 1 & Homoz. & 1.0 & Homoz. & Homoz. \\
\hline 2 & 0.98 & 0.92 & 1.0 & Homoz. \\
\hline 3 & 0.91 & 0.91 & 0.83 & 1.0 \\
\hline 4 & 1.0 & Homoz. & 1.0 & 1.0 \\
\hline 5 & 0.94 & 0.78 & 0.23 & Homoz. \\
\hline 6 & 1.0 & 0.91 & 0.83 & Homoz. \\
\hline 7 & Homoz. & Homoz. & Homoz. & Homoz. \\
\hline 8 & 0.98 & 1.0 & 0.91 & 1.0 \\
\hline 9 & 0.96 & 0.91 & 0.91 & Homoz. \\
\hline 10 & 1.0 & 1.0 & Homoz. & Homoz. \\
\hline 11 & Homoz. & Homoz. & 0.91 & 0.83 \\
\hline 12 & 0.97 & 0.77 & 0.91 & Homoz. \\
\hline 13 & 0.96 & 1.0 & 1.0 & Homoz. \\
\hline 14 & 0.96 & 1.0 & Homoz. & 1.0 \\
\hline 15 & Homoz. & Homoz. & 1.0 & 0.82 \\
\hline 16 & 0.95 & Homoz. & 0.83 & 0.91 \\
\hline 17 & 0.96 & 0.91 & Homoz. & Homoz. \\
\hline 18 & Homoz. & Homoz. & Homoz. & Homoz. \\
\hline 19 & Homoz. & 0.35 & 0.0 & 0.23 \\
\hline 20 & 1.0 & Homoz. & 1.0 & 0.83 \\
\hline 21 & 0.34 & 0.28 & 0.21 & Homoz. \\
\hline 22 & Homoz. & 1.0 & 0.62 & 0.55 \\
\hline 23 & Homoz. & 0.91 & 0.23 & 0.83 \\
\hline 24 & 0.99 & Homoz. & Homoz. & 0.91 \\
\hline 25 & 1.0 & 0.83 & Homoz. & Homoz. \\
\hline 26 & 0.99 & 1.0 & 0.78 & Homoz. \\
\hline 27 & 1.0 & 1.0 & 0.83 & Homoz. \\
\hline 28 & Homoz. & 1.0 & 1.0 & Homoz. \\
\hline 29 & 1.0 & 0.99 & 0.92 & 0.91 \\
\hline 30 & 0.94 & Homoz. & 0.92 & Homoz. \\
\hline 31 & Homoz. & 0.0 & 0.37 & 0.0 \\
\hline 32 & 0.94 & Homoz. & 0.83 & Homoz. \\
\hline 33 & 1.0 & 0.91 & 0.83 & 0.77 \\
\hline 34 & 0.98 & 0.71 & Homoz. & Homoz. \\
\hline 35 & 0.94 & 0.83 & 0.91 & 0.97 \\
\hline 36 & Homoz. & 1.0 & 0.68 & Homoz. \\
\hline 37 & 0.97 & 1.0 & Homoz. & Homoz. \\
\hline 38 & 0.93 & 0.91 & 1.0 & 0.91 \\
\hline 39 & Homoz. & 0.91 & 0.91 & 0.99 \\
\hline 40 & Homoz. & 0.85 & 0.91 & 1.0 \\
\hline 41 & Homoz. & Homoz. & 1.0 & Homoz. \\
\hline 42 & Homoz. & 0.91 & 0.91 & Homoz. \\
\hline 43 & 0.45 & Homoz. & 0.86 & 1.0 \\
\hline 44 & 1.0 & 0.91 & 0.83 & 1.0 \\
\hline
\end{tabular}

Homoz. $=$ homozygosity. Numbers in bold $=Q$ (peak area) $\leq 0.50$ indicating $\mathrm{LOH}$. mal karyotype by G-banding presented $\mathrm{LOH}$. It was for the marker D7S515. Otherwise, FISH centromeric probe for chromosome 7 showed monosomy in $25 \%$ of the cells. The 2 patients with complex karyotype (cases 21 and 43) presented LOH for IRF-1. The karyotype of case 21 was $47, X X,+8[1] / 47, X X, \operatorname{del}(5)(q 13 q 33), \operatorname{del}(7)(q 22),+8[2] /$ $49, X X, \operatorname{del}(7)(q 22),+8,+11,+18[1]$ and case 43 was $53-$ $56, X X,+1,+8,+9,+10$, i $(11)(q 13),+14,+15,+16,+17,+18$, $+19, \sim 6 \mathrm{dmin}$.

It is important to note that there was a significantly positive association of the occurrence of $\mathrm{LOH}$ with abnormal karyotype ( $P<0.05$; chi-square test).

$\mathrm{LOH}$ on chromosome $7 \mathrm{q}$ has been frequently reported in several types of human cancer including myeloid neoplasia (1). Using a panel of three microsatellite markers at band $7 q 31$, we found 5 of $44(11 \%)$ patients with one or more allelic loss. Among these 5 cases, three presented cytogenetic abnormalities involving chromosome 7 (cases 5,19 , and 21). Case 5 presented monosomy 7 by FISH and LOH for D7S515; case 19 showed monosomy 7 and LOH of the three markers, as expected; case 21 presented $\mathrm{LOH}$ of D7S486 and D7S515, but the deletion was at band 7q22. Koike et al. (11) studied the LOH on the long arm of chromosome 7 in AML patients using a panel of 15 markers and found a higher incidence of $\mathrm{LOH}(27 \%)$ than in the present study probably due to the different panel with more markers (fifteen). In fact, Basirico et al. (12) studied the $\mathrm{LOH}$ of $7 q$ using a panel of 11 polymorphic microsatellite markers at band 7q21-36 and correlated the findings with conventional karyotype. Sixteen patients (16/50) showed allelic loss, but only three presented cytogenetic abnormalities related to chromosome 7 . Our results suggest that $\mathrm{LOH}$ in different microsatellite markers on the long arm of chromosome 7 is an event previous to chromosomal abnormalities in these myeloid neoplasias, with karyotype abnormalities occurring later.

An important finding among the present cases was the correlation between LOH of IRF-1 and complex karyotype with a lot of trisomies, deletions, isochromosome and double minutes concomitantly. An interesting aspect that remains to be determined is if $I R F-1$ gene expression is decreased in these cases of $\mathrm{LOH}$. Unfortunately, immunohistochemistry for IRF-1 performed in these cases was not successful, and there were no more samples available for additional molecular studies. Notwithstanding, IRF-1 has been implicated as a mediator for interferon signaling, when induced by TNF- $\alpha$, interferons, viral infections and retinoids. IRF-1 has been considered to be a TSG that plays an essential role in cell growth control and surveillance against malignant development (13). The findings of the present study support the view of IRF-1 as an important TSG. 


\section{References}

1. Loeb LA, Springgate CF, Battula N. Errors in DNA replication as a basis of malignant changes. Cancer Res 1974; 34: 2311-2321.

2. Krskova-Honzatkova L, Cermak J, Sajdova J, Stary J, Sedlacek P, Sieglova Z. Loss of heterozygosity and heterogeneity of its appearance and persisting in the course of acute myeloid leukemia and myelodysplastic syndromes. Leuk Res 2001; 25: 45-53.

3. List AF, Vardiman J, Issa JP, DeWitte TM. Myelodysplastic syndromes. Hematol Am Soc Hematol Educ Program 2004; 297-317.

4. Chauffaille ML, Yamamoto M, Moncau JE, Braga GW, Souto EX, Kerbauy J. Cytogenetic abnormalities as prognostic factors in acute myeloid leukemia. Rev Assoc Med Bras 1996; 42: 200-204.

5. Shaffer LG, Tommerup N (Editors). ISCN (2005): An international system for human cytogenetic nomenclature. Basel: S. Karger Publishers; 2005.

6. Greenberg P, Cox C, LeBeau MM, Fenaux P, Morel P, Sanz $\mathrm{G}$, et al. International scoring system for evaluating prognosis in myelodysplastic syndromes. Blood 1997; 89: 20792088.

7. Grimwade D, Walker $\mathrm{H}$, Oliver $\mathrm{F}$, Wheatley $\mathrm{K}$, Harrison $\mathrm{C}$, Harrison G, et al. The importance of diagnostic cytogenetics on outcome in AML: analysis of 1,612 patients entered into the MRC AML 10 trial. The Medical Research Council Adult and Children's Leukaemia Working Parties. Blood 1998; 92:
2322-2333.

8. Harris NL, Jaffe ES, Diebold J, Flandrin G, Muller-Hermelink HK, Vardiman J, et al. World Health Organization classification of neoplastic diseases of the hematopoietic and lymphoid tissues: report of the Clinical Advisory Committee Meeting - Airlie House, Virginia, November 1997. J Clin Oncol 1999; 17: 3835-3849.

9. Lee TH, Sakahara NS, Fiebig EW, Hirschkorn DF, Johnson DK, Busch MP. Quantitation of white cell subpopulations by polymerase chain reaction using frozen whole-blood samples. Viral Activation Transfusion Study. Transfusion 1998; 38: 262-270.

10. Cawkwell L, Bell SM, Lewis FA, Dixon MF, Taylor GR, Quirke P. Rapid detection of allele loss in colorectal tumours using microsatellites and fluorescent DNA technology. $\mathrm{Br} \mathrm{J}$ Cancer 1993; 67: 1262-1267.

11. Koike M, Tasaka T, Spira S, Tsuruoka N, Koeffler HP. Allelotyping of acute myelogenous leukemia: loss of heterozygosity at 7q31.1 (D7S486) and q33-34 (D7S498, D7S505). Leuk Res 1999; 23: 307-310.

12. Basirico R, Pirrotta R, Fabbiano F, Mirto S, Cascio L, Pagano $M$, et al. Submicroscopic deletions at $7 q$ region are associated with recurrent chromosome abnormalities in acute leukemia. Haematologica 2003; 88: 429-437.

13. Liebermann DA, Hoffman B. Myeloid differentiation (MyD) primary response genes in hematopoiesis. Oncogene 2002; 21: 3391-3402. 